\title{
Electromyography-Guided Botulinum Toxin Injection Into the Cricothyroid Muscles in Bilateral Vocal Fold Abductor Paralysis
}

\author{
Mustafa Sahin ${ }^{1} \cdot$ Ibrahim Aydogdu² $\cdot$ Serdar Akyildiz ${ }^{3} \cdot$ Munevver Erdinc $^{4} \cdot$ Kerem Ozturk $^{3} \cdot$ Fatih Ogut $^{3}$ \\ ${ }^{1}$ Department of Otolaryngology, Adnan Menderes University Medical School, Aydin; \\ Departments of ${ }^{2}$ Neurology, ${ }^{3}$ Otolaryngology, and ${ }^{4}$ Chest Diseases, Ege University Medical School, Izmir, Turkey
}

Objectives. Bilateral vocal fold abductor paralysis (BVFAP) both deteriorates quality of life and may cause life-threatening respiratory problems. The aim of this study was to reduce respiratory symptoms in BVFAP patients using cricothyroid (CT) botulinum toxin (BTX) injection.

Methods. Before and 2 weeks and 4 months after bilateral BTX injection into the CT muscles under electromyography; alterations in respiratory, acoustic, aerodynamic and quality of life parameters were evaluated in BVFAP patients with respiratory distress. For the respiratory evaluation modified Borg scale and spirometry, for the voice and aerodynamic evaluations Voice Handicap Index-30 (VHI-30), GRBAS, acoustic analysis (sound pressure level, F0, jitter\%, shimmer\%, noise-to-harmonic ratio) and maximum phonation time and for the quality of life assessment Short Form-36 (SF-36) form were used.

Results. All patients were female with a mean age of $47 \pm 8.1$ years. There was a mean time of $11.8 \pm 5.5$ (minimum 2, maximum 23) months between BVFAP development and BTX injection. In all cases, other than one case with unknown aetiology, the cause of vocal fold paralysis was prior thyroid surgery. In total 18.6 \pm 3.1 units of BTX were applied to the CTs. In the preinjection period, and the 2nd week and 4th month after injection, the Borg dyspnea scale was 7.3/5.3/5.0, FIV1 (forced inspiratory volume in one second) was 1.7/1.7/1.8 L, peak expiratory flow (PEF) was 1.4/1.7/2.1 L/sec, maximum phonation time was 7.0/6.4/6.2 seconds and VHI-30 was 63.2/52.2/61.7 respectively. There was no significant alteration in acoustic analysis parameters. Many of the patients reported transient dysphagia within the first week. There were insignificant increases in SF-36 sub-scale values.

Conclusion. After BTX injection, improvements in the mean Borg score, PEF and FIV1 values and SF-36 sub-scale scores showed the restricted success of this approach. This modality may be kept in mind as a transient treatment option for patients refused persistent tracheotomy or ablative airway surgeries.

Keywords. BilateralVocal Fold Paralysis; Botulinum Toxin; Cricothyroid Muscle

\section{INTRODUCTION}

Since restriction of vocal fold (VF) movements generally takes place due to inferior laryngeal nerve dysfunction, this functional

- Received February 4, 2016

Revised March 30, 2016

Accepted April 9, 2016

- Corresponding author: Mustafa Sahin

Department of Otorhinolaryngology, Adnan Menderes University Medical

School, 09100, Aydin, Turkey

Tel: +90-533-654-5502, Fax: +90-256-218-2000

E-mail: iskebaha@gmail.com deterioration is referred to as 'VF paralysis' in general practice. Restriction of VF movements is generally of the abductor paralysis type [1]. The cause of VF paralysis is commonly iatrogenic surgical damage especially due to thyroidectomy [2]. Bilateral vocal fold abductor paralysis (BVFAP) results in a pathological period of highly deteriorating quality of life, causing respiratory compromise and increased risk of tracheotomy. Patients should restrict their daily activities in order not to accelerate their respiratory compromise [3]. The main aim of BVFAP treatment is to restore adequate ventilation. If the inferior laryngeal nerve defect is not absolute, patients should be followed-up for a period

Copyright (C) 2017 by Korean Society of Otorhinolaryngology-Head and Neck Surgery.

This is an open-access article distributed under the terms of the Creative Commons Attribution Non-Commercial License (http://creativecommons.org/licenses/by-nc/4.0)

which permits unrestricted non-commercial use, distribution, and reproduction in any medium, provided the original work is properly cited. 
to monitor the recovery of VF movements. During this period of time, irreversible or destructive surgical procedures such as arytenoidectomy or cordotomy should be avoided. There is still not an optimal treatment method to reduce respiratory distress in this conservation period. Tracheotomy may be required to control the increased dyspnea triggered by respiratory system infections, increased physical activity or weight gain. At the end of this period (almost one year), some surgical procedures (cordotomy, arytenoidectomy, lateralization of VF by suture, type II thyroplasty) are advised to reduce the airway failure in patients in whom abductor functions were not reversed by spontaneous regeneration and respiratory distress was not improved $[3,4]$. These surgical procedures generally affect the voice undesirably and may increase the aspiration risk during swallowing. Although tracheotomy is highly effective in the control of respiratory distress, it has some risks such as tracheal stenosis and infection and moreover it leads to a very unpleasant cosmetic defect, a significant deterioration in quality of life and psychosocial impairment $[4,5]$. However, laryngeal re-innervation and electrical pulse stimulation protocols are not yet a viable alternative in the treatment of VF paralysis [6].

Botulinum toxin (BTX) inhibits acetylcholine secretion in neuronal ends of the neuromuscular junction and leads to chemical denervation. It does not cause any morphological alteration or cellular death in nerve endings. BTX has been demonstrated clinically to be an effective treatment for a variety of laryngeal problems such as cricopharyngeal spasm, VF granuloma, laryngeal tremor, paradoxical VF motion, and pharyngoesophageal hypertonicity in patients with voice prosthesis after laryngectomy [7]. Application of the toxin results in a flask paralysis in the affected muscle for 3-6 months. Data about the usage of BTX in BVFAP in laryngology especially in spasmodic diseases of the larynx is limited. However, BTX injection into different internal laryngeal muscle groups has been reported in some studies of BVFAP to prevent aberrant re-innervation and synkinesis and to widen the airway [6-8].

In early phases of paralysis, more lateral position of VFs may result in moderate dyspnea and accompanying breathy voice and aspiration. In time, the balance of adductor/abductor mus-

\section{H}

- Respiration and voice were measured in 25 patients with bilateral vocal fold abductor paralysis before and after botulinum toxin injection to bilateral criothyroid muscles.

- Respiratory and voice measurements after botulinum injection showed limited success.

- Twenty-four patients suffering respiratory problems were evaluated before and 2 weeks and 4 months after bilateral injection.

- The transient treatment might be optional for patients who refuse tracheostomy or ablative airway surgery. cle powers with especially chronic unopposed activity of intact cricothyroid (CT) muscles which has the function of stretching VFs to the anteromedially could potentially result in gradual more medial positioning of the VFs in BVFAP [9]. Unbalanced adductor powers reinforce gradual narrowing of the airway results in an escalation in respiratory distress with a relative improvement in voice quality $[9,10]$. The typical mid-line position of the resting VFs' tonus has been attributed to the development of a synkinesis with adductor dominancy $[1,9,10]$. Neuromuscular blockade of synkynetic reinnervated adductor muscles may be beneficial in some patients with BVFAP.The use of BTX in VF paralysis is associated with the hypothesis of blocking dominancy of adductor muscles. Eliminating the function of CT muscle produces gradual lateral bowing of the effected VF [11]. The rationale for chemical denervation of the CT muscles is to reduce the tension of the VF thus to change the position of the VF to a more lateral position. In an animal study of Cohen and Thompson BTX was injected into the CT muscles to attain lateralizastion. They demonstrated that paralysis of the CT muscles decreased the tension of the VFs and allows them to take a more lateral position and thereby improve the airway [12]. Since then, BTX injection to intralaryngeal muscles has been considered as a possible therapy for the airway compromise in patients with BVFAP. BTX can be used to block inappropriate synkinesis that has developed in the laryngeal adductor muscles, and thus increase the glottal airway [10]. Ekbom et al. [13] and Daniel and Cordona [14] proposed intralaryngeal BTX injection to be considered in the treatment algorithm for BVFAP as temporizing therapy to avoid emergent surgical management.

The aim of this study was to investigate the scientific validity of the hypothesis in a larger patient group that 'chemical denervation of CT muscles with BTX-type A (BTXA) diminishes dyspnea in patients with BVFAP by changing the positioning of the VFs.' One of the main primary challenge while managing BVFAP is to perform a procedure that improves the airway but still maintains a serviceable voice because most of the surgical procedures described irreversibly sacrifice voice in order to improve airway [10,12]. The second aim of this study was to assess voice change after BTXA injection as a less invasive method. In view of this aims; respiratory, acoustic, aerodynamic and life quality parameters were evaluated in BVFAP patients with respiratory distress, before and after BTXA injection bilaterally into the CT muscle under electromyography (EMG).

\section{MATERIALS AND METHODS}

\section{Patients}

This multidisciplinary study was carried out in a university hospital and it was approved by the Clinical Investigations Ethics Committee of university hospital (B.30.2.EGE.0.21.05.00/ EY/304-136) as retrospective case series. The included patients 
were selected from patients admitted to our clinic with respiratory distress and diagnosed with BVFAP following a physical examination. Surgical treatments were initially explained and advised in patients having paralysis for more than one year. The patients were informed that the effectiveness of BTX treatment in bilateral VF paralysis is not yet clear. The patients were told that the obtained benefit would be transient and recurrent BTX injections may be required. Patients in the re-innervation period and patients with more than one year of diagnosis and denied persistent surgical treatment were included in the study. The exclusion criteria were as follows: under 18 years old, having comorbid diseases that can cause respiratory distress, having any neurological disease that can result in respiratory distress or muscle involvement, presence of any neuromuscular disease history such as myasthenia gravis or myopathies, pregnant and lactating women, history of psychiatric diseases, laryngeal surgery or trauma, or radiotherapy to head and neck region, development of VF paralysis in a malign disease course, allergy to BTX, having paralysis other than the abductor type, having a main symptom other than dyspnea, and having a modified Borg dyspnea score (MBS) of less than 4 at the initial evaluation.

In all included patients, a BTXA (Allergan, Irvine, Ireland) injection was administered bilaterally to the $\mathrm{CT}$ muscles, under EMG guidance. After injection, patients were followed in clinic for a period of time (almost four hours) and then discharged with the advice that they should return if they experienced any increase in respiratory distress. Alterations in respiratory, acoustic, aerodynamic and life quality parameters were evaluated in all patients, before and in the 2nd week and 4th month after BTXA injection. Moreover, patients were asked to record possible laryngopharyngeal problems (such as dysphagia, aspiration, breathy phonation, and pain) until their next examination. The patients evaluated their own dysphagia (if present) grade using a visual analogue scale (VAS).

\section{LEMG and BTXA injection procedure}

Laryngeal EMG (LEMG) was performed using a two-channel EMG machine (Medelec Synergy; Oxford Instruments, Surrey, UK) while the patient was in a semi-recumbent/sitting position with the head tilted posteriorly. A disposable concentric needle EMG electrode (Ambu Neuroline Inoject, 26G, Ballerup, Denmark) was used for both EMG evaluation and BTXA injection of the CT muscles. The needle was inserted percutaneously near the superior border of the cricoid cartilage in the posterolateral direction and directed superiorly along the lateral border of the thyroid cartilage. The patient was asked to phonate a strong high-pitched /i/ to enable correct CT muscle insertion and was also asked to tense the neck and tilt the head anteriorly to help avoid he neck strap muscles. After verification of needle placement, EMG activity was evaluated at rest (for denervation) and during high-pitched phonation (for motor unit evaluation during voluntary activity). Features of denervation potentials and mo- tor unit potentials such as normal/neurogenic were noted. Then BTXA was applied to the CT muscles bilaterally with the same electrode using EMG guidance.

\section{Respiratory evaluation}

Though the MBS is currently generally used to define the severity of exertion dyspnea, it can also be used to evaluate the severity of resting dyspnea. It provides the clinician with quick and easy data about the subjective dyspnea condition of the patient. It is formed from 10 points that describe dyspnea severity: 0 (no dyspnea) and 10 (the most severe dyspnea experienced ever) [15]. Each case pointed out their dyspnea level on this scale before and in the 2nd week and 4th month after BTXA injection. Patients with tracheotomy evaluated their condition with their cannula closed.

Spirometry was performed (Vmax encore PFT system, Care Fusion, San Diego, CA, USA) in all cases during each evaluation (except in patients with a tracheotomy cannula), and forced inspiratory volume in one second (FIV1) in litres (L), forced vital capacity (FVC) in L, forced expiratory volume in one second (FEV1) in L, FEV1/FVC ratio, and peak expiratory flow (PEF) in $\mathrm{L} / \mathrm{sec}$ were also evaluated.

\section{Otolaryngological examination, and voice and acoustic evaluation}

All patients had a detailed otolaryngological and neurological physical examination before and after BTXA injection. Videolaryngostroboscopic examination (Xion Endo-Strob DX, Berlin, Germany) was performed by the same otolaryngology specialist who was blinded to the patients. There was no evidence of upper airway infection during recording in any of the patients.

Self-assessment of voice quality was scored using the Turkish version of the Voice Handicap Index 30 (VHI-30). VHI-30 is a questionnaire composed of 30 questions. Subjects score $0-4$ for each question. Higher scores infer increased problems [16].

Perceptual voice quality was evaluated using the GRBAS scale by an experienced specialist who did not know the study design or patients. GRBAS is a reliable and valid scale consisting of five parameters (grade, roughness, breathiness, asthenia, strain) and is universally used in auditory-perceptual evaluation of voice quality. For each parameter, four different scores from 0 to 3 are given according to the severity of dysphonia ( 0 is normal, 1 is slight degree, 2 is medium degree, and 3 is high degree of severity) [17]. GRBAS scoring was performed by an assessor using samples of a reading passage in Turkish that comprised 219 words with rich and balanced phonemes.

Voice samples were recorded in a sound-insulated room at a sound level at which the patients felt relaxed, while seated upright, using a high quality omnidirectional microphone (Shure SM48, Niles, IL, USA) placed approximately $15 \mathrm{~cm}$ from the lips. The subject was instructed to phonate the vowel /a/ at a habitual pitch and comfortable loudness for 5 seconds. Patients 
with tracheostomy were instructed to close the cannula during phonation and speaking. Each patient was given a short practice period prior to the first recording to adapt to the procedure. The task was repeated three times to yield three examples of vowel /a/ for each subject. Each trial was captured on a hard disk at a $44,100-\mathrm{Hz}$ sampling rate and a 16-bit resolution. Computerized Speech Lab (Kay PENTAX CSL model 4500, Montvale, NJ, USA) software (CSL main programme and MDVP) was used to capture and analyse the voice samples. The first and last second of each sample were discarded to avoid unintended irregularities and variability on voicing onset and offset. The mean values were calculated for each subject. Five parameters of the voice analyses were examined: (1) fundamental frequency (F0) in $\mathrm{Hz}$, (2) sound pressure level (SPL) in $\mathrm{dB},(3)$ jitter\% (Jitt) as a frequency perturbation parameter, (4) shimmer\% (Shim) as an amplitude perturbation parameter, and (5) noise-to-harmonic ratio (NHR) as a spectral parameter.

\section{Aerodynamic evaluation}

Maximum phonation time (MPT) was calculated as the duration of vowel $\backslash \mathrm{a} \backslash$ after maximum inspiration. The longest time of three different measurements was regarded as the MPT.

\section{Quality of life evaluation}

The Short Form-36 (SF-36) life quality scale, developed by Ware and Sherbourne in 1992, is a universal measurement method with previous validity and reliability studies that evaluates life quality in a general manner and is not specific for any disease. It can be performed easily in a short time and has a very broad application. SF-36 is formed from 36 questions in eight subscales (general health, physical function, physical role, emotional role, social function, pain, energy, mental health). Each scale is scored from 0 to 100 . The lower the score the worse the quality of life [18]. The quality of life of each case was evaluated by each patient before and in the 2nd week and 4th month after BTXA injection.

\section{Statistical analysis}

All data obtained in the study were analysed using the IBM SPSS ver. 20.0 (IBM Co., Armonk, NY, USA). Changes in patients' data over time were evaluated using a paired sample $t$ test. Since the data from patients who achieved unilateral VF motion significantly affected the results, these patients were evaluated separately as group I. Changes in six patients who experienced a unilateral correction in paralysis (group I) were evaluated using the Wilcoxon signed-ranked test while changes in 24 patients who did not show any improvement in paralysis (group II) were evaluated using a paired sample $t$-test. To investigate differences between patients who did or did not have any unilateral improvement in paralysis, the Mann-Whitney $U$-test was performed. The numerical results are presented as means. Statistical significance was set at $P<0.05$.

\section{RESULTS}

All 24 patients demonstrated voluntary electrophysiological activity of the CT muscles on EMG. Three patients who did not have any CT muscle denervation in EMG were excluded from the study and BTXA injection was not given to these patients. Two of the patients who received BTXA injection did not attend the follow-up examinations and were thus excluded. Four patients had a tracheotomy cannula. All 24 included patients were female with a mean age of $47 \pm 8.1$ years. The mean time between BVFAP development and BTXA application was 11.8土 5.5 months and the total BTXA dose applied to both CT muscles of patients was $18.6 \pm 3.1 \mathrm{U}$. Twelve of the 24 patients were in their first year after BVFAP diagnosis when the injection was administered (mean 6.6, min 2, max 12 months). In all cases, other than one case with an unidentified etiology, the cause of VF paralysis was preceding total thyroidectomy surgery.

All patients attended the 2nd week follow-up but three patients missed the 4th month follow-up. In the 2nd week after injection, VF motion was not observed in any of the cases. In the 4th month unilateral VF motion was observed in five patients. Moreover, unilateral VF motion was observed in one patient who attended the 6-month but not the 4-month follow-up (giving a total of $6 / 24$ with unilateral VF remobility). One of these six patients had a tracheostomy cannula and after the resumption of VF mobility she was decannulated. Since the data from patients who achieved unilateral VF mobility were significantly different and since their results affected the data of patients overall, these two groups were separated for further analysis. Patients who achieved unilateral VF mobility after injection were defined as group I, while those patients without any improvements in BVFAP were defined as group II. Group II (patients with ongoing BVFAP) were split into another two groups according alteration in MBS score: patients who benefitted from BTXA injection (at least two point decrease in MBS score, group IIa) and patients who did not benefit (group IIb). Table 1 summarizes the general characteristics and results of the statistical comparison of group I and II. Age, applied BTXA dose, respiratory, acoustic, aerodynamic and life quality parameters before BTXA injection did not differ significantly between group I and II or between group IIa and IIb. The period of time between BVFAP development and BTXA injection was significantly shorter

Table 1. Comparison of age, duration of BVFAP and BTX injection dose according to recovery of unilateral vocal fold movement

\begin{tabular}{lccc}
\hline Variable & Group I $(n=6)$ & Group II $(n=18)$ & $P$-value \\
\hline Age $(\mathrm{yr})$ & $45.0 \pm 5.2$ & $48.0 \pm 8.8$ & 0.594 \\
Duration of BVFAP (mo) & $5.0 \pm 2.9$ & $13.1 \pm 4.7$ & 0.001 \\
BTX dose $(\mathrm{U})$ & $18.5 \pm 3.9$ & $18.6 \pm 3.0$ & 0.762
\end{tabular}

Values are presented as mean \pm SD. Statistical significance was set at $P<0.05$

BVFAP, bilateral vocal fold abductor paralysis; BTX, botulinum toxin. 
in group I (5.0 vs. 13.1 months, $P=0.001)$.

The MBS and spirometry results of patients before and in the 2nd week and 4th month after BTXA injection are summarized in Table 2. In all patients, there were statistically significant differences in MBS, FIV1, and PEF values in the 2nd week and 4th month compared with the pre-BTXA injection values (decrease in MBS, increase in FIV1 and PEF). In group I, the 4th month results of these parameters differed significantly from both the preinjection and 2nd week results. There were no significant differences in the other parameters (FEV1, FVC, FEV1/FVC) over time (for group I between preinjection and 2nd week results, for group II between all three periods). The 4th month results of group I differed significantly from the preinjection and 2nd week results. Changes in the other parameters over time (for group I between preinjection and 2nd week results, for group II between all three periods) were not statistically significant.

The perceptual evaluation of voice quality and acoustic analysis of voice parameters prior to and two weeks and four months after the BTXA injection are presented in Table 3. The VHI-30, GRBAS values and measured acoustic analysis parameters did not change significantly during the study and did not differ between groups. When patients in group I were evaluated in more

Table 2. Parameters related to MBS and PFT values prior to and 2 weeks and 4 months after BTX-type A injection

\begin{tabular}{|c|c|c|c|c|c|c|}
\hline \multirow{2}{*}{ Parameter } & \multicolumn{3}{|c|}{ Group I $(n=6)^{a)}$} & \multicolumn{3}{|c|}{ Group II $(n=18)^{b)}$} \\
\hline & Pre-BTX & $2 w k$ & $4 \mathrm{mo}$ & Pre-BTX & $2 w k$ & $4 \mathrm{mo}$ \\
\hline MBS & $7.8 \pm 1.4$ & $5.2 \pm 1.5$ & $2.3 \pm 1.8$ & $7.6 \pm 0.8$ & $6.2 \pm 0.9$ & $6.0 \pm 1.0$ \\
\hline FIV1 (L) & $1.5 \pm 0.1$ & $1.7 \pm 0.2$ & $2.2 \pm 0.3$ & $1.5 \pm 0.1$ & $1.6 \pm 0.2$ & $1.8 \pm 0.2$ \\
\hline FEV1 (L) & $1.6 \pm 0.2$ & $1.9 \pm 0.4$ & $2.3 \pm 0.3$ & $1.6 \pm 0.3$ & $1.6 \pm 0.3$ & $1.6 \pm 0.3$ \\
\hline FVC (L) & $1.9 \pm 0.1$ & $2.0 \pm 0.1$ & $2.4 \pm 0.3$ & $1.8 \pm 0.8$ & $1.8 \pm 0.1$ & $1.9 \pm 0.4$ \\
\hline FEV1/FVC & $66.6 \pm 8.3$ & $68.5 \pm 5.6$ & $78.1 \pm 6.4$ & $64.5 \pm 6.4$ & $66.1 \pm 5.2$ & $67.3 \pm 4.1$ \\
\hline PEF (L/sec) & $1.4 \pm 0.5$ & $1.7 \pm 1.1$ & $3.6 \pm 1.1$ & $1.5 \pm 0.4$ & $1.7 \pm 0.3$ & $1.7 \pm 0.2$ \\
\hline
\end{tabular}

Values are presented as mean $\pm \mathrm{SD}$.

MBS, modified Borg scale; PFT, pulmonary function test; BTX, botulinum toxin; FIV1, forced inspiratory volume in one second; FEV1, forced expiratory volume in one second; FVC, forced vital capacity; PEF, peak expiratory flow.

a) One patient with tracheostomy did not perform spirometry. ${ }^{b}$ One patient with tracheotomy did not perform spirometry.

Table 3. Parameters related to perceptual evaluation and acoustic analysis prior to and 2 weeks and 4 months after BTX-type A injection

\begin{tabular}{|c|c|c|c|c|c|c|}
\hline \multirow{2}{*}{ Parameter } & \multicolumn{3}{|c|}{ Group I $(n=6)$} & \multicolumn{3}{|c|}{ Group II $(n=18)$} \\
\hline & Pre-BTX & $2 w k$ & $4 \mathrm{mo}$ & Pre-BTX & $2 w k$ & $4 \mathrm{mo}$ \\
\hline VHI-30 & $66.6 \pm 7.7$ & $54.6 \pm 8.5$ & $62.3 \pm 8.4$ & $62.1 \pm 10.7$ & $58.5 \pm 9.9$ & $61.5 \pm 8.8$ \\
\hline GRBAS & $5.5 \pm 0.8$ & $5.4 \pm 0.5$ & $6.1 \pm 1.7$ & $5.4 \pm 1.0$ & $5.8 \pm 0.6$ & $5.3 \pm 0.6$ \\
\hline $\mathrm{MPT}^{\star}(\mathrm{sec})$ & $6.8 \pm 1.2$ & $6.5 \pm 1.2$ & $4.8 \pm 1.0$ & $7.1 \pm 1.0$ & $6.3 \pm 0.8$ & $6.2 \pm 0.9$ \\
\hline $\mathrm{FO}(\mathrm{Hz})$ & $215 \pm 16$ & $199 \pm 45$ & $201 \pm 30$ & $227 \pm 27$ & $233 \pm 31$ & $228 \pm 23$ \\
\hline $\mathrm{SPL}(\mathrm{dB})$ & $57.6 \pm 2.3$ & $54.5 \pm 1.8$ & $50.1 \pm 3.1$ & $55.3 \pm 3.7$ & $54.1 \pm 2.7$ & $53.9 \pm 3.1$ \\
\hline Jitter\% & $1.3 \pm 0.3$ & $1.5 \pm 0.6$ & $1.8 \pm 0.3$ & $1.4 \pm 0.7$ & $1.4 \pm 0.5$ & $1.5 \pm 0.6$ \\
\hline Shimmer\% & $3.2 \pm 1.4$ & $3.6 \pm 1.6$ & $3.8 \pm 1.6$ & $3.7 \pm 1.0$ & $3.9 \pm 1.5$ & $3.9 \pm 1.1$ \\
\hline NHR & $0.17 \pm 0.05$ & $0.16 \pm 0.05$ & $0.19 \pm 0.03$ & $0.17 \pm 0.05$ & $0.15 \pm 0.03$ & $0.16 \pm 0.03$ \\
\hline
\end{tabular}

Values are presented as mean $\pm \mathrm{SD}$.

BTX, botulinum toxin; VHI, voice handicap index; GRBAS, grade, roughness, breathiness, asthenia, strain; MPT, maximum phonation time (*as an aerodynamic evaluation parameter); FO, mean fundamental frequency; SPL, sound pressure level; NHR, noise-to-harmonic ratio.

Table 4. Values for each sub-scale of SF-36 survey prior to and 2 weeks and 4 months after BTX-type A injection

\begin{tabular}{|c|c|c|c|c|c|c|}
\hline \multirow{2}{*}{ Variable } & \multicolumn{3}{|c|}{ Group I $(n=6)$} & \multicolumn{3}{|c|}{ Group II $(n=18)$} \\
\hline & Pre-BTX & $2 w k$ & $4 \mathrm{mo}$ & Pre-BTX & $2 w k$ & $4 \mathrm{mo}$ \\
\hline Physical functioning & $48.6 \pm 3.7$ & $52.6 \pm 5.5$ & $80.3 \pm 4.6$ & $52.1 \pm 5.7$ & $58.5 \pm 5.9$ & $61.5 \pm 4.8$ \\
\hline Role-physical & $48.5 \pm 2.8$ & $55.4 \pm 8.5$ & $81.1 \pm 6.7$ & $53.4 \pm 2.0$ & $55.8 \pm 3.6$ & $56.3 \pm 3.7$ \\
\hline Bodily pain & $66.8 \pm 2.2$ & $66.5 \pm 4.2$ & $76.0 \pm 3.0$ & $67.1 \pm 2.1$ & $69.4 \pm 3.8$ & $68.3 \pm 4.9$ \\
\hline General health & $34.6 \pm 1.6$ & $42.3 \pm 4.5$ & $82 \pm 4.2$ & $36.1 \pm 3.2$ & $41.5 \pm 6.9$ & $42.5 \pm 5.7$ \\
\hline Vitality & $33.4 \pm 2.3$ & $37.8 \pm 6.8$ & $79.1 \pm 7.1$ & $35.3 \pm 4.4$ & $44.1 \pm 5.7$ & $43.9 \pm 6.1$ \\
\hline Social functioning & $37.3 \pm 4.0$ & $43.3 \pm 4.5$ & $77.8 \pm 5.2$ & $39.4 \pm 5.6$ & $41.1 \pm 4.5$ & $40.3 \pm 4.6$ \\
\hline Role-emotional & $41.3 \pm 3.7$ & $52.1 \pm 6.4$ & $79.2 \pm 6.7$ & $40.1 \pm 3.6$ & $42.3 \pm 4.1$ & $41.4 \pm 5.3$ \\
\hline Mental health & $39.7 \pm 4.6$ & $56.1 \pm 5.3$ & $76.8 \pm 6.6$ & $37.7 \pm 3.2$ & $41.2 \pm 2.1$ & $39.4 \pm 0.0$ \\
\hline
\end{tabular}

SF-36, Short Form-36; BTX, botulinum toxin. 
Table 5. Comparison of aerodynamic and voice-related parameters of group Ila and Ilb before and after BTX-type A injection

\begin{tabular}{lccccc}
\hline \multirow{2}{*}{ Parameter } & \multicolumn{2}{c}{ Group $\| \mathrm{la}(\mathrm{n}=5)$} & & \multicolumn{2}{c}{ Group $\| \mathrm{lb}(\mathrm{n}=13)$} \\
\cline { 2 - 3 } \cline { 5 - 6 } & Pre-BTX & 4th mo & & Pre-BTX & 4th mo \\
\hline MBS & $7.7 \pm 0.9$ & $5.3 \pm 1.4$ & & $7.6 \pm 0.6$ & $6.9 \pm 1.1$ \\
FIV1 (L) & $1.5 \pm 0.1$ & $1.9 \pm 0.2$ & & $1.5 \pm 0.2$ & $1.7 \pm 0.1$ \\
FVC $(\mathrm{L})$ & $1.8 \pm 0.2$ & $1.9 \pm 0.1$ & & $1.8 \pm 0.2$ & $1.9 \pm 0.2$ \\
FEV1 $(\mathrm{L})$ & $1.6 \pm 0.3$ & $1.7 \pm 0.2$ & & $1.6 \pm 0.3$ & $1.6 \pm 0.3$ \\
PEF $($ L/sec $)$ & $1.5 \pm 0.4$ & $1.8 \pm 1.1$ & & $1.5 \pm 0.5$ & $1.6 \pm 0.3$ \\
VHI & $63.4 \pm 8.2$ & $62.7 \pm 9.1$ & & $61.2 \pm 7.9$ & $63 \pm 7.2$ \\
GRBAS & $5.5 \pm 1.2$ & $5.1 \pm 0.7$ & & $5.4 \pm 0.8$ & $5.5 \pm 0.6$ \\
MPT (sec) & $7.3 \pm 1.3$ & $6.0 \pm 1.1$ & & $7.0 \pm 1.2$ & $6.4 \pm 1.4$ \\
F0 (Hz) & $228 \pm 33$ & $229 \pm 31$ & & $224 \pm 28$ & $226 \pm 26$ \\
Jitter\% & $1.4 \pm 0.7$ & $1.5 \pm 0.5$ & & $1.4 \pm 0.5$ & $1.5 \pm 0.4$ \\
Shimmer\% & $3.6 \pm 1.3$ & $3.9 \pm 1.5$ & & $3.7 \pm 1.4$ & $3.9 \pm 1.3$ \\
NHR & $0.16 \pm 0.02$ & $0.17 \pm 0.03$ & & $0.17 \pm 0.04$ & $0.15 \pm 0.01$ \\
\hline
\end{tabular}

Values are expressed as mean \pm SD.

BTXA, botulinum toxin type A; MBS, modified Borg scale; FIV1, forced inspiratory volume in one second; FVC, forced vital capacity; FEV1, forced expiratory volume in one second; PEF, peak expiratory flow; VHI, voice handicap index; GRBAS, grade, roughness, breathiness, asthenia, strain; MPT, maximum phonation time; FO, mean fundamental frequency; NHR, noise-to-harmonic ratio.

detail in terms of GRBAS scores in the 4th month, the ' $\mathrm{S}$ ' parameter was found to have decreased, but the ' $G$ ' and especially 'B' parameters had increased and thus the total score had increased.The MPT value of group I decreased insignificantly over time. On the other hand, group II showed a significant reduction in MPT values in the 2nd week and 4th month compared with the preinjection values.

The SF-36 life quality evaluation parameters prior to and 2 weeks and 4 months after BTXA injection are presented in Table 4. On all scale scores, group I showed an insignificant increase in the 2nd week and a significant increase after 4 months compared with the other periods. In group II, there were increases in all SF-36 sub-scale scores in the 2nd week and 4th month, but the differences between time periods were not statistically significant.

When group II (18 patients with ongoing BVFAP) were analysed in detail there was a reduction in the MBS score and increase in some spirometry values (FIV1, FVC, and PEF) and total SF-36 score at the 4-month evaluation. MBS, aerodynamic and voice evaluation parameters in group IIa and IIb before and in the 4th month after BTXA injection are summarized in Table 5. There were significant increases in FIV1 and mean SF-36 score in patients who showed no improvement in MBS (group $\mathrm{IIb}, 13$ patients) in the 4th month after BTXA injection and insignificant increases in PVF and PEF. There was a statistically significant decrease in MBS score and increase in FIV1, FVC, and PEF values and the total SF-36 score in group IIa (five patients). Laryngoscopic evaluation pictures of a patient from group IIa before and after the bilateral CT muscle BTX injection
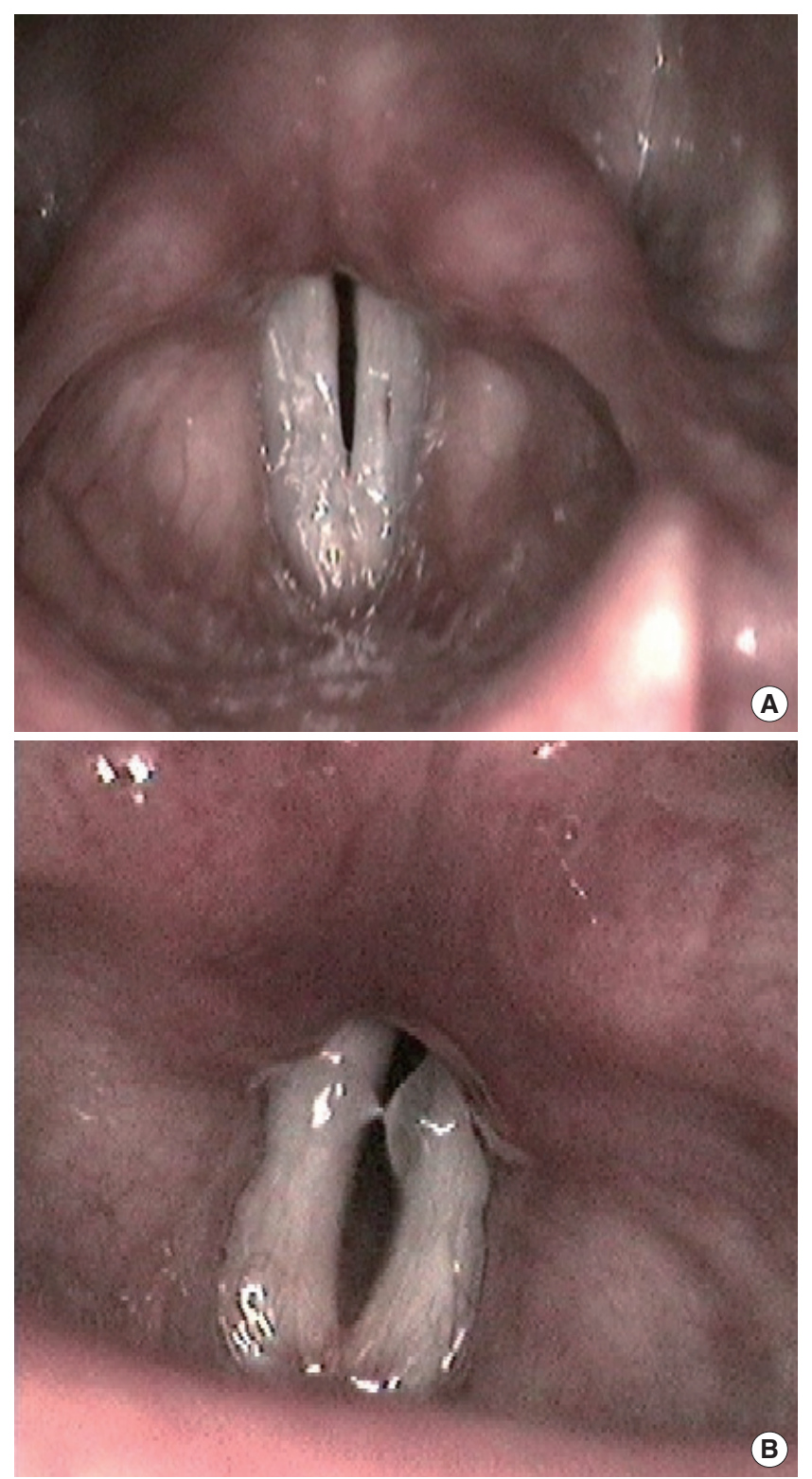

Fig. 1. Laryngoscopic evaluation pictures of a patient with bilateral vocal fold abductor paralysis during maximum abduction before $(A)$ and after $(B)$ the bilateral cricothyroid muscle bilateral botulinum toxin injection.

was submitted in Fig. 1.

In brief, in this study there were improvements in symptoms associated with dyspnea (decrease in MBS score) and quality of life (increase in SF-36 sub-scale scores) without any deterioration in voice quality 4 months after BTXA injection. These results were consistent with some objective pulmonary function test results (increase in FIV1 and PEF) and voice analysis parameters (Fig. 2).

Two of the patients who reported an improvement in respiratory distress after injection requested a repeat injection at 5 months and one patient requested it at 6 months due to recur- 


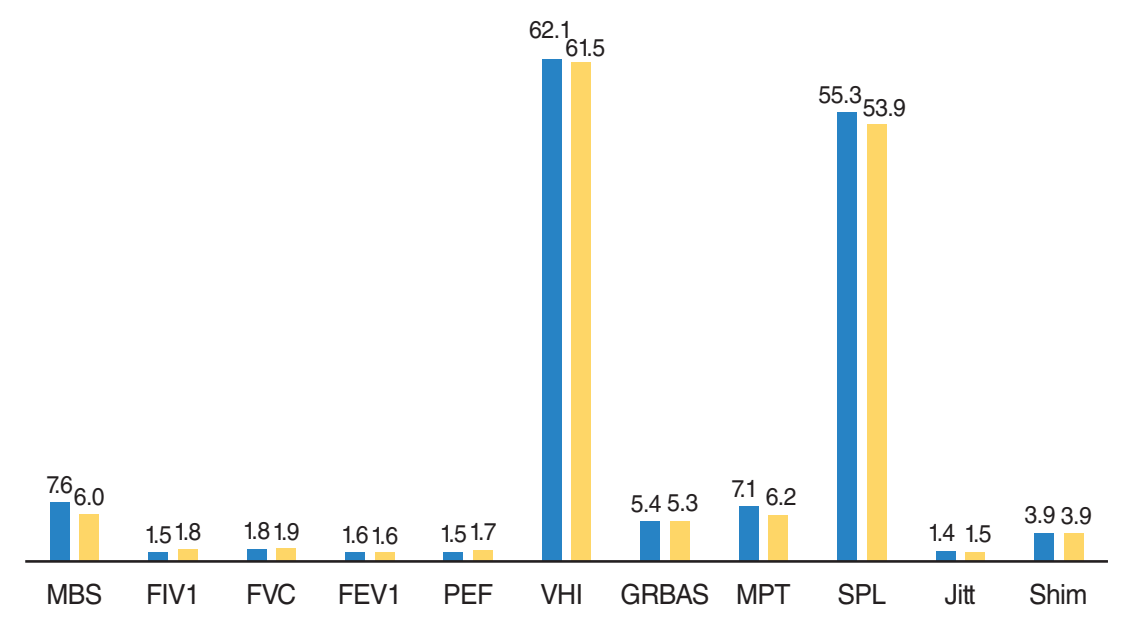

Fig. 2. Comparison of aerodynamic and acoustic analysis parameters of group II before (first column) and 4 months after botulinum toxin type $A$ injection (second column). MBS, modified Borg scale; FIV1, forced inspiratory volume in one second; FVC, forced vital capacity; FEV1, forced expiratory volume in one second; PEF, peak expiratory flow; $\mathrm{VHI}$, voice handicap index; GRBAS, grade, roughness, breathiness, asthenia, strain; MPT, maximum phonation time; SPL, sound pressure level; Jitt, jitter; Shim, shimmer. rence of their symptoms. Bilateral BTXA injection into the CT muscle was thus repeated in these three patients. Two of these patients had a tracheotomy, and approximately 1 month after the second injection were decannulated. Posterior laser cordotomy was performed in two of the patients who did not show any improvements at any of the follow-up appointments. One of them had a tracheotomy and was decannulated after cordotomy.

The mean VAS dysphagia score of patients in the month prior to the BTXA injection was $0.8 \pm 0.3$ while it was $3.4 \pm 1.2$ in the 2 nd week and $0.9 \pm 0.3$ between 2 weeks and 4 months after injection.

\section{DISCUSSION}

BVFAP has an extensive morbiditiy. The search for simpler and better method to rehabilitate the BVFAP has been an elusive and persistent issue for otolaryngologists. There is still not an optimal treatment option for BVFAP. The surgical methods to treat BVFAP has been controversial and sometimes disappointing [12]. The use of BTX to relief airway symptoms in BVFAP is also controversial and is not common and has been sparsely documented in the clinical literature $[7,10]$. Biochemical denervation of CT muscles may diminish the tonus in the laryngeal adductor and rebalance of the forces within the intralaryngeal musculature. To the best of our knowledge, this is the largest study in terms of patient number and evaluated parameters to evaluate effectiveness of BTX injection administered to both CT muscles in BVFAP under EMG.

In many of the BVFAP cases dyspnea is the main symptom. The other most important symptom is usually dysphonia. Due to the gap between the VFs, voice quality may be better than in the case of unilateral VF paralysis, but a monotonous voice and speaking pattern characterized by short and hurried words followed by stridor of frequent frequency spectrum is not rare [19]. Dysphagia, aspiration and pain in the neck region may also be seen [3]. In our study, the primary symptom was respiratory dis- tress and the secondary symptom was dysphonia. Patients with a tracheotomy reported that they wanted to get rid of their cannulas as soon as possible. The patients did not report any dysphagia in the 2 months prior to BTX injection.

The activity of BTX is measured in mouse units (MU, LD50, $\mathrm{U})$. Although there is no exact dose regimen for laryngeal BTX application, bilateral applications of $2.5 \mathrm{U}$ for each side and unilateral applications of 5-15 U BTXA are commonly advised for spasmodic dysphonia. It has been reported that the exact BTXA dose can be determined after the second application [20]. In this study, the BTXA dose was 9 units for each CT muscle. This dose is higher than the doses used elsewhere. We thought that we would use higher BTXA doses due to the presence of paralysis and since we were injecting into the CT muscle.

BTX could be applied bilaterally or unilaterally to the thyroarytenoid muscle, TA/LCA muscle complex and CT muscles to relief airway compromise in BVFAP. Laryngeal BTX applications could be performed transcutaneously under EMG, with the help of transnasal fibreoptic endoscopy, or with direct vision under general anaesthesia $[9,11]$. In this study, BTX injection was performed under EMG in all patients without anesthesia, with no complications being observed during the injections or at subsequent follow-up. CT muscles were easily reached using the external approach. For this reason, this application method was regarded as safe, rapid and can be used widely.

Unlike the other internal laryngeal muscles, the CT muscle is innervated by the external branch of the superior laryngeal nerve. It approximates the cricoid ring to the lower border of the thyroid cartilage and it effects elevation and deviation of the cricoids ring toward the stimulated side thereby produces tension, elongation and adduction of the VFs $[13,21]$. In paralytic $\mathrm{VF}$, endogenous abductor power may result in more manifest adductor effects of the CT muscle. As the paralysis proceeds, the unopposed effect of the CT muscle may result in more severe airway problems [8,9]. In an animal study by Cohen et al. [22], chemical CT muscle denervation with BTX for bilateral VF paralysis was reported to produce VF lateralization without any 
complications or histological damage in muscles. In this study, we applied BTXA to CT muscles in order to produce an improvement in the airway by decreasing the adductor effect and the tension of the VFs. Daniel and Cordona [14] reported onabotulinum toxin A injection of the CT muscles in BVFAP as a safe, rapid, effective, and non-invasive option. Benninger et al. [9] considered BTXA injection of the CT muscles may prevent progression to airway compromise requiring more invasive therapy in their study. In studies that aimed to reduce laryngeal adductor tonus via BTX injection into the thyroarytenoid/lateral cricoarytenoid (TA/LCA) muscle complex, restricted and transient improvements were reported in respiration. Ekbom et al. [13] administered BTX injection to the TA/LCA complex of 11 patients and observed a partial improvement in subjective respiratory symptoms. Smith et al. [23] applied bilateral BTX injection to the thyroarytenoid muscles (and additionally to the CT muscles in two children) of 10 children with respiratory distress and bilateral VF paralysis under general anesthesia and reported a significant improvement in respiratory distress in four patients. Another reason for preferring the CT muscles in this approach was our inadequate experience in EMG of the TA/LCA muscle complex. Earlier injections may achieve more significant improvement. This may be associated with the trend for re-innervation through the abductor muscles. In our study, we achieved greater improvement in symptoms when the BTX injection was performed earlier after onset of paralysis. In this regard, our results are consistent with those of Ekbom et al. [13].

After BTX injection into the CT muscle bilaterally, Benninger et al. [9] reported an improvement in respiratory distress of three patients. In our study we measured a subjective (a decrease in Borg score after injection) improvement in respiratory distress of patients after injection. This improvement was also apparent in FIV1 and PEF. In fact the exact mechanism of this condition is not known but it has been suggested that chemical denervation of active $\mathrm{CT}$ muscles may produce flaccidity in the VFs (Fig. 1) and cause a minimal increase in glottic section area $[9,18,19]$. This increase, though minimal, may improve respiratory distress even in patients with life-threatening levels of distress.

Daniel and Cordona [14] offered a trial of BTX injection of the CT muscles may save the patient from multiple intubations and avert the need for a tracheostomy. In our study the tolerance of decannulation by two patients was very encouraging. However, patients must be aware that the improvement in respiration is temporary, lasting approximately $3-5$ months, and, if BTX is chosen as a method of management, then repeat or serial injections will be required [11,13]. Because 4 to 12 weeks after BTX application, new nerve terminals are produced via nerve sprouting and neural messages begin through the muscle. In this way the effectiveness of BTX starts to decline. The mean time required for the re-start of neuromuscular transmission for BTXA is approximately 3 months $[7,10]$. Three of the six patients in group II who showed a more obvious benefit from BTX injection (decrease in Borg score of at least 3 points) reported that their symptoms recurred at the end of the 5th month and injection was repeated in those patients. This suggests that the effects of BTX had begun to diminish. Andrade Filho and Rosen [11] reported a successful management of a patient with BVFAP by repetitive injections of BTX. Cohen et al. [22] reported that multiple injections did not cause irrecoverable damage to the laryngeal muscles. Prolonged effects can be achieved by multiple injections [12]. This results are promising for the patients who refuse surgical methods and also for the patients who is in the earlier period of paralysis because these group of patients may require repetetive BTX injections. Three of our patients were received second BTX injection after the initial success. In order to evaluate this period (decreasing effect of BTX) more objectively and clearly EMG could be performed intermittently. The lack of such EMG controls may be regarded as a limitation of this study. Moreover, more data should be acquired to determine the effectiveness of BTX over repeated applications. Although it is a simple and painless procedure, the main disadvantage of BTX injection to $\mathrm{CT}$ is the requirement for repeated injections. Moreover, resistance may develop in some patients, although this is rare. An insufficient dose, the mass of the muscle, patient age, technique applied, unfavorable synkinesis, and not complying with the cold chain procedure may affect the success of BTXA injection [24].

When evaluating the limitations of patients due to dyspnea during routine daily activities, the statistically significant decrease in the MBS after BTX injection and the trend, though not statistically significant, for an increase in quality of life assessment scales were encouraging results showing the subjective success of injection in this study. Evaluation of the pulmonary function test results showed an increase in FIV1, FEV1, and PEF values in the 4th month. A significant increase in those parameters in group IIa, which showed improvements in upper airway obstruction, was a promising result. Group IIb patients who showed no significant relief in their symptoms had increased FIV1 (significant), PEF, and FEV1 values, an increased total SF36 score and a decreased MBS score. This was also a promising but paradoxical result that needs to be addressed in future studies. There was no statistically significant change in group II patients' voice outcome results (VHI, GRBAS, SPL, Jitt, Shim, NHR) after BTXA injection. This was also a favoring result about the voice outcome of this procedure.

Although the voice is less affected in BVFAP compared with unilateral paralysis, the voice quality of patients usually deteriorates (weak, tense, intermittent, and exerted) compared with the preoperative period. The high VHI-30 scores of our patients at admission show that the patients were dissatisfied with their voice quality. The GRBAS values were consistent with these findings. Moreover, in the acoustic analysis and determination of Jitt, Shim, and NHR, the higher than normative values reported in the literature were evaluated as subjective roughness and deterioration in voice [25]. After surgical treatment of BV- 
FAP there is no improvement in dyspnea without a decrease in voice quality. Benninger et al. [9] reported a transient cough, aspiration and worsening in voice quality in a patient after bilateral BTX injection into the CT. There were no significant changes in self-evaluation of voice, perceptual voice quality assessment or acoustic analysis parameters in the 2nd week and 4th month after BTXA injection, and this was regarded as an advantage since there was no undesirable effect on the voice.

An main advantages of the BTX injection are (1) is not a permanent effect so patients who received this procedure have not lost other therapeutic options, (2) does not endamage normal anatomy, (3) takes its effect almost immediately, (4) has no systemic toxicity when injected small local area $[9,17,22]$. In our study posterior laser cordotomy was performed in two of the patients who did not show any improvements after BTX injection.

The main side effects reported after laryngeal BTX application are dysphonia with breathy voice, dysphagia, aspiration of low-density fluids and itchiness in the throat. No severe side effects of laryngeal BTX injection have been reported and the most common local side effect is dysphagia $[9,26]$. In general, this dysphagia decreases until it disappears within a few days. In our study, globus sensation in the throat, dysphagia and coughing during drinking that was thought to be due to aspiration, were reported in the first five days after injection. For this reason, we recommend informing patients about safe swallowing techniques in the first week after BTX injection in order to prevent possible food aspiration during that period.

The main weaknesses of this study were: (1) all patients were female, although this was a coincidence and not part of the study design; (2) the etiology was thyroidectomy in almost all patients, which makes our results specific for this group of patients. Studies are warranted in patient groups with other BVFAP etiologies. Ongkasuwan and Courey [10] reported that the most appropriate patient group for adductor chemical denervation was patients with inferior laryngeal nerve paralysis such as that due to surgical trauma; (3) TA/LCA muscle EMG to exclude cricoarytenoid joint fixation could be added but our patients' medical histories were not appropriate for this pathology; (4) long-term follow-up of patients with recurrent dose application were lacking; a decrease in dyspnea was shown by subjective data (patient reports and endoscopic appearance), but not by the application of intermittent electrophysiological evaluations (EMG). Moreover, with the application of BTX to other muscles, especially to the TA/LCA muscle complex, comparisons would be possible. Studies with larger patient populations and with BTX applications under EMG to the different muscle groups are warranted.

In conclusion, following BTXA injection into the CT muscles, only limited benefits were observed despite improvements in respiratory distress as reported by patients in objective evaluations. No distinct deterioration in voice quality was recorded. Patients in whom paralysis had lasted one year or less and who had normal EMG results for the bilateral CT muscles were thought to be the most likely to benefit from bilateral CT muscle BTXA injection. Otolaryngologists should be' aware that some patients may prefer BTXA injection to persistent tracheotomy or destructive airway surgery. The easy application of a BTXA injection to the CT muscles bilaterally should be considered as a possible temporary solution for such patients with symptomatic airway compromise to avoid emergent surgical interventions. Bilateral CT BTXA injection would offer transient relief of dyspnea to patients in whom the prognosis of VF mobility recovery remains unclear. Moreover, these patients would not lose the opportunity to benefit from other treatment options.

\section{CONFLICT OF INTEREST}

No potential conflict of interest relevant to this article was reported.

\section{REFERENCES}

1. Zealear DL, Billante CR. Neurophysiology of vocal fold paralysis. Otolaryngol Clin North Am. 2004 Feb;37(1):1-23.

2. Myssiorek D. Recurrent laryngeal nerve paralysis: anatomy and etiology. Otolaryngol Clin North Am. 2004 Feb;37(1):25-44.

3. Misiolek M,Waler J, Namyslowski G, Kucharzewski M, PodwinskiA, Czecior E. Recurrent laryngeal nerve palsy after thyroid cancer surgery: a laryngological and surgical problem. Eur Arch Otorhinolaryngol. 2001 Nov;258(9):460-2.

4. Hillel AD, Benninger M, Blitzer A, Crumley R, Flint P, Kashima HK, et al. Evaluation and management of bilateral vocal cord immobility. Otolaryngol Head Neck Surg. 1999 Dec;121(6):760-5.

5. Dursun G, Gokcan MK. Aerodynamic, acoustic and functional results of posterior transverse laser cordotomy for bilateral abductor vocal fold paralysis. J Laryngol Otol. 2006 Apr;120(4):282-8.

6. Misono S, Merati AL. Evidence-based practice: evaluation and management of unilateral vocal fold paralysis. Otolaryngol Clin North Am. 2012 Oct;45(5):1083-108.

7. Blitzer A, Sulica L. Botulinum toxin: basic science and clinical uses in otolaryngology. Laryngoscope. $2001 \mathrm{Feb} ; 111(2)$ :218-26.

8. Zalvan $\mathrm{CH}$, Blitzer A. Using botulinum toxin therapy in the laryngopharynx. Oper Tech Otolaryngol Head Neck Surg. 2004 Jun;15(2): 86-9.

9. Benninger MS, Hanick A, Hicks DM. Cricothyroid muscle botulinum toxin injection to improve airway for bilateral recurrent laryngeal nerve paralysis, a case series. JVoice. 2016 Jan;30(1):96-9.

10. Ongkasuwan J, Courey M. The role of botulinum toxin in the management of airway compromise due to bilateral vocal fold paralysis. Curr Opin Otolaryngol Head Neck Surg. 2011 Dec;19(6):444-8.

11. Andrade Filho PA, Rosen CA. Bilateral vocal fold paralysis: an unusual treatment with botulinum toxin. JVoice. 2004 Jun;18(2):254-5.

12. Cohen SR, Thompson JW. Use of botulinum toxin to lateralize true vocal cords: a biochemical method to relieve bilateral abducto $\mathrm{r}$ vocal cord paralysis. Ann Otol Rhinol Laryngol. 1987 Sep-Oct;96(5): 534-41.

13. Ekbom DC, Garrett CG, Yung KC, Johnson FL, Billante CR, Zealear $\mathrm{DL}$, et al. Botulinum toxin injections for new onset bilateral vocal fold motion impairment in adults. Laryngoscope. 2010 Apr;120(4): 
758-63.

14. Daniel SJ, Cardona I. Cricothyroid onabotulinum toxin A injection to avert tracheostomy in bilateral vocal fold paralysis. JAMA Otolaryngol Head Neck Surg. 2014 Sep;140(9):867-9.

15. Mahler DA, Horowitz MB. Clinical evaluation of exertional dyspnea. Clin Chest Med. 1994 Jun;15(2):259-69.

16. Kilic MA, Okur E, Yildirim I, Ogut F, Denizoglu I, Kizilay A, et al. Reliability and validity of the Turkish version of the Voice Handicap Index. Kulak Burun Bogaz Ihtis Derg. 2008 May-Jun;18(3):139-47.

17. Eadie T, Sroka A, Wright DR, Merati A. Does knowledge of medical diagnosis bias auditory-perceptual judgments of dysphonia? J Voice. 2011 Jul;25(4):420-9.

18. Ware JE Jr, Sherbourne CD. The MOS 36-item short-form health survey (SF-36). I. Conceptual framework and item selection. Med Care. 1992 Jun;30(6):473-83.

19. Yilmaz T, Suslu N, Atay G, Ozer S, Gunaydın RO, Bajin MD. Comparison of voice and swallowing parameters after endoscopic total and partial arytenoidectomy for bilateral abductor vocal fold paralysis: a randomized trial. JAMA Otolaryngol Head Neck Surg. 2013 Jul;139(7):712-8.

20. Holden PK, Vokes DE, Taylor MB, Till JA, Crumley RL. Long-term botulinum toxin dose consistency for treatment of adductor spas- modic dysphonia. Ann Otol Rhinol Laryngol. 2007 Dec;116(12): 891-6.

21. Merati AL, Halum SL, Smith TL. Diagnostic testing for vocal fold paralysis: survey of practice and evidence-based medicine review. Laryngoscope. 2006 Sep;116(9):1539-52.

22. Cohen SR, Thompson JW, Camilon FS Jr. Botulinum toxin for relief of bilateral abductor paralysis of the larynx: histologic study in an animal model.Ann Otol Rhinol Laryngol. 1989 Mar;98(3):213-6.

23. Smith ME, Park AH, Muntz HR, Gray SD. Airway augmentation and maintenance through laryngeal chemodenervation in children with impaired vocal fold mobility. Arch Otolaryngol Head Neck Surg. 2007 Jun;133(6):610-2

24. Niamtu J 3rd. Botulinum toxin A: a review of 1,085 oral and maxillofacial patient treatments. J Oral Maxillofac Surg. 2003 Mar;61(3): 317-24.

25. Tatar EC, Sahin M, Demiral D, Bayir O, Saylam G, Ozdek A, et al. Normative values of voice analysis parameters with respect to menstrual cycle in healthy adult Turkish women. J Voice. 2016 May;30 (3):322-8.

26. Neuenschwander MC, Pribitkin EA, Sataloff RT. Botulinum toxin in otolaryngology. In: Sataloff RT, editor. Treatment of voice disorders. San Diego: Plural Publishing Inc.; 2005. p. 167-77. 